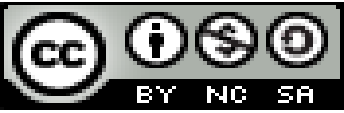

Ciencias económicas y empresariales

Artículo de investigación

\title{
Análisis correlacional entre la producción del aceite de palma, sus exportaciones y su contribución al PIB agrícola durante el período 2010 - 2017
}

Correlational analysis between the production of palm oil, its exports and its contribution to agricultural GDP during the period 2010 - 2017

\section{Análise correlacional entre a produção de óleo de palma, suas exportações e sua contribuição para o PIB agrícola no período de 2010 a 2017}

\author{
Mauro C. Tapia-Toral I \\ mauro.tapiato@ug.edu.ec
}

Fredy G. Alvarado-Espinoza II

freddyalvaradorp@hotmail.com

Recibido: 23 de junio de 2018 * Corregido: 14 de agosto de 2018 * Aceptado: 17 de septiembre de 2018

I. Magister en Finanzas y Proyectos Corporativos, Economista con Mención en Gestión Empresarial Especialización Finanzas, Contador Público, Docente de la Universidad de Guayaquil, Guayaquil, Ecuador.

II. Magister en Negocios Internacionales y Gestión en Comercio Exterior, Economista, Docente de la Universidad de Guayaquil, Guayaquil, Ecuador. 


\section{Resumen}

En la presente investigación se tiene como objetivo analizar el comportamiento del Producto interno bruto frente a la producción del aceite de palma y sus exportaciones. Se realiza un análisis de descriptivo correlacional de tipo transversal - analítico, cuya línea de investigación se enfoca al estudio de la economía, desarrollo Local y regional. Los resultados obtenidos confirman la relación directa fuerte en la producción de palma y una relación directa débil en las exportaciones con respecto al producto interno bruto agrícola. Lo cual determina que el potencial agrícola en el sector palmicultor donde por cada dólar invertido se obtiene un beneficio directo de 0.87 .

Palabras clave: aceite de palma; producción agrícola; economía agrícola; producto interno bruto.

\section{Abstract}

In the present investigation, the objective is to analyze the behavior of the gross domestic product against the production of palm oil and its exports. A correlational descriptive analysis of transversal analytical type is carried out, whose research line focuses on the study of economics, Local and regional development. The results obtained confirm the strong direct relationship in palm production and a weak direct relation in exports with respect to agricultural gross domestic product. Which determines that the agricultural potential in the palm oil sector where for every dollar invested a direct benefit of 0.87 is obtained.

Key words: palm oil; agricultural production; agricultural economy; gross domestic product.

\section{Resumo}

Na presente investigação, o objetivo é analisar o comportamento do produto interno bruto em relação à produção de óleo de palma e suas exportações. É realizada uma análise descritiva correlacional do tipo analítico - transversal, cuja linha de pesquisa enfoca o estudo da economia, desenvolvimento local e regional. Os resultados obtidos confirmam a forte relação direta na produção de palma e uma fraca relação direta nas exportações em relação ao produto interno bruto agrícola. Que determina que o potencial agrícola no setor de óleo de palma, onde para cada dólar investido, um benefício direto de 0,87 é obtido.

Palavras chave: óleo de palma; produção agrícola; economia agrícola; produto interno bruto. 


\section{Introducción}

El aceite de palma, posee una amplia variedad de usos como son: la elaboración de jabones de aseo personal, elaboración de cosméticos, procesamiento de aceites de origen vegetal, productos de limpieza, base para la elaboración de balanceados, entre otros. Lo que constituye una importante materia prima en el sector comercial e industrial. Se obtiene de la extracción del fruto de la Palma aceitera conocida comúnmente como palma africana.

Las especificaciones del suelo para el desarrollo de estos tipos de cultivos al de una textura francolimoso a franco-arcilloso, cuyas superficies posean una amplia porosidad y un rango de pH de 5 a 6.5 (Asociación Nacional de Cultivadores de Palma Aceitera, 2003: 9) y cuyas condiciones climáticas sean de amplia precipitación de entre 1800 y 2000 milímetros con humedad relativa del 75\%, y climas cálidos que oscilen entre los 21 y 28 grados centígrados

Debido a los niveles de rentabilidad ofrecidos por la venta del aceite de palma y en particular los costos de procesamiento para la elaboración de los derivados que involucran la importación de materiales para el proceso de transformación a los derivados del aceite de palma. El estado ha debido de participar como mediador respecto a los niveles de importaciones de los insumos y sustitutos. (Carrión y Cuvi, 1985: 56).

El sector económico dedicado a la investigación y experimentación para obtener mejoras derivados del aceite, han generado espacios de aceptación en el mercado mundial. Se analiza la evolución de la producción la materia prima, la palma africana, las exportaciones del mismo, así como la producción del aceite que se obtiene de la palma africana, y el Producto interno bruto no petrolero.

Países de clima tropical como Malasia e Indonesia que poseen las condiciones favorables para este tipo de cultivo poseen alrededor del $80 \%$ de la producción internacional en el sector, por lo que el precio internacional depende de las condiciones de oferta y demanda que generen, así también el aceite de soya interviene en el comportamiento del aceite de palma. El Ecuador genera una producción promedio anual de 500 mil toneladas métricas del producto y en su participación se acogen a las condiciones del mercado en el que participa. (Vallejo, 2012) 
Factores exógenos ocurridos en el 2010 al 2017 ha afectado las exportaciones del aceite de palma en el Ecuador, generándose un incremento en la producción de soya, lo cual también ha afectado al precio del aceite de palma (MAGAP, 2014).

El análisis de la relación de la industria del aceite de palma ante la dependencia de los precios internacionales, y en los bienes de la cadena de valor hacia arriba de sus productos subsiguientes determina los siguientes factores:

La participación del mercado ecuatoriano en el sector internacional, equivale alrededor del 1\%, por tal motivo, la producción nacional del aceite de palma se debe ajustar a los precios determinados por los demás entes internacionales. Siendo así que países como Indonesia y Malasia poseen alrededor del $80 \%$ de la producción mundial, lo cual les faculta la posibilidad de influenciar en el precio internacional.

Un sustituto del aceite de palma constituye el aceite de soya, y su producción afecta de manera directa a los precios nacionales. Estos acontecimientos generan variaciones principalmente en las exportaciones nacionales del producto y sus derivados, principalmente ante caídas en los precios internacionales del bien a exportar. Así también las fluctuaciones del precio del aceite de soya.

Como (Yánez, 2013) manifiesta que el Ecuador se caracteriza por ser un proveedor de recursos naturales o materia prima. Encabezando la producción petrolera, seguido bienes tradicionales de producción agrícola como el banano, cacao, café, flores, de producción marítima como el camarón, atún, sardinas que comprenden los principales rubros de generación de divisas para el estado, además expone que la agricultura provee alrededor del 40\% de las plazas ocupacionales de la población económicamente activa, sin considerar de la producción destinada al consumo interno. Frente a esta dinámica se relaciona que la economía ecuatoriana basa como uno de sus principales rubros la producción agrícola, y dentro de ello participa la producción del aceite de palma.

En el territorio ecuatoriano se genera una producción de la semilla que provee el aceite de palma en alrededor de 17,8 toneladas por hectárea, siendo este componente la base para la elaboración de diversos usos y elaboración de productos de consumo masivo, al ser un antioxidante de origen natural rico en betacarotenos y tocotrienoles equivalentes a la vitamina $\mathrm{A}$ y E, su uso también se extiende a la producción de biocombustibles por su alto valor energético. (PRO ECUADOR, 2014). 
Cifras provenientes del Ministerio de Comercio Exterior, 2018, indican que el aceite de palma constituye un importante rubro de exportación, llegando a ocupar el séptimo lugar dentro de los rubros agrícolas, y contribuye al dinamismo de la industria no tradicional, así como también en la producción no petrolera.

Todo ello remarca en la importancia de la producción del aceite de palma y el potencial e importancia que provee para el desarrollo sustentable de la economía ecuatoriana, así como la generación de divisas por medio de las exportaciones de sus derivados.

\section{Material y Métodos}

Para el estudio de la presente investigación, se analizarán el comportamiento de las exportaciones de aceite de palma del Ecuador, así como también los factores que contribuyen al desarrollo del PIB Agrícola ecuatoriano para el período 2010 al 2017. La información proporcionada por el Banco Central del Ecuador (BCE), constituye la base para realizar el análisis cuantitativo, así como también los resultados de las encuestas realizadas por Instituto Nacional de Estadísticas y Censos (INEC) respecto a la producción, exportación de la palma africana y aceite de palma.

\section{Tipo de Estudio}

Para el desarrollo de los procedimientos establecidos en el presente trabajo de investigación, a fin de obtener las conclusiones sobre el objeto de estudio. Se utiliza una metodología de tipo cuantitativo descriptivo de corte transversal 2010 al 2017 para identificar las características a través de los datos estadísticos relacionados al comportamiento de las exportaciones del aceite de palma en el Ecuador.

\section{Diseño de Contrastación de Hipótesis.}

Se realiza un diseño de tipo no experimental, descriptivo, debido a que no existen grupos experimentales o de control que describan el comportamiento de los hechos. Siendo además que las evoluciones de los precios del aceite de palma influyen a los rubros de exportación, y estos rubros contribuyen al PIB agrícola se plantea que las exportaciones del aceite de palma africano contribuyen de manera significativa al producto interno agrícola del Ecuador. 


\section{Población Muestra y Muestreo}

Para el presente estudio se utiliza como población la superficie plantada del territorio ecuatoriano, los niveles de rendimiento por área cosechada su producción y exportaciones del aceite de palma dentro del territorio ecuatoriano.

\section{Métodos, Técnicas e Instrumentos de Recolección de Datos}

El tratamiento de los datos es descriptivo, y correlacional debido a que se analizan las relaciones que existen entre las variables y estudia en términos de ingresos y generación de recursos que contribuyen al desarrollo productivo agrícola e industrial nacional, por medio de uso del análisis correlacional para describir el comportamiento de la realidad nacional.

\section{Variables de estudio}

Para medir el efecto correlacional sobre el PIB agrícola, se utilizó los rubros de, las exportaciones del aceite de Palma (XP) que hace referencia a los excedentes de producción nacional; la producción del aceite de palma (PA) obtenida de la producción nacional; y el Producto interno agrícola (PIB).

\section{Procesamiento y Análisis de Datos}

Las técnicas e instrumentos a utilizar en la presente investigación consisten en recopilar la información generada por las cuentas nacionales proporcionada por el BCE e INEC tabuladas por Eviews 6.0 a través de un análisis correlacional lineal, para medir los comportamientos entre las exportaciones, y producción del aceite de palma en su contribución al PIB agrícola ecuatoriano.

El coeficiente de correlación lineal es una medida que analiza la relación lineal entre dos variables cuantitativas (David Anderson, 2012), es decir, la medida de relación de dos variables, definida por la ecuación:

$\mathrm{rx}, \mathrm{y}=\mathrm{Sx}, \mathrm{y} /(\mathrm{SxSy})$ 
Con lo cual, al enumerar todos los coeficientes de correlación simples de un conjunto de variables, se forma la matriz de correlación, misma que incluye las correlaciones de las variables analizadas. (Douglas Lind, 2012).

\section{Matriz de correlaciones}

\begin{tabular}{|c|c|c|c|}
\hline & $\begin{array}{l}\text { Exportaciones } \\
\text { de aceite de } \\
\text { palma }(X P)\end{array}$ & $\begin{array}{c}\text { Producción } \\
\text { de aceite } \\
\text { de palma } \\
\text { (PA) }\end{array}$ & $\begin{array}{c}\text { PIB } \\
\text { Agrícola }\end{array}$ \\
\hline $\mathrm{XP}$ & 1 & & \\
\hline PA & 0.408 & 1 & \\
\hline PIB Agrícola & 0.160 & 0.879 & 1 \\
\hline
\end{tabular}

Fuente. Banco Central del Ecuador

\section{Resultados y Discusión}

\section{Evolución de las exportaciones y producción del aceite de palma}

En Latinoamérica el Ecuador se sitúa en el segundo lugar de producción del aceite de palma, cultivándose en las provincias Costeras: Esmeraldas, Santo Domingo, Guayas, Los Ríos, Manabí, de la serranía: Cotopaxi y Bolívar, y de la zona oriental: Orellana y Sucumbíos. Poseen condiciones favorables para la producción de la planta que provee el aceite de palma.

Este producto es considerado de alta importancia para el sector agropecuario, debido a que contribuye en un 4,53\% y su producción se destina principalmente al consumo interno y su desarrollo se ha incrementado paulatinamente, siendo así que por cada dólar destinado a la producción agrícola se obtiene un impacto positivo de 0.40 en las exportaciones no tradicionales del sector agrícola.

Se observa un crecimiento sostenido desde el 2013 en los niveles de producción del aceite de palma, mientras que las exportaciones a partir del 2014 su crecimiento se ha mantenido de manera sostenida. 


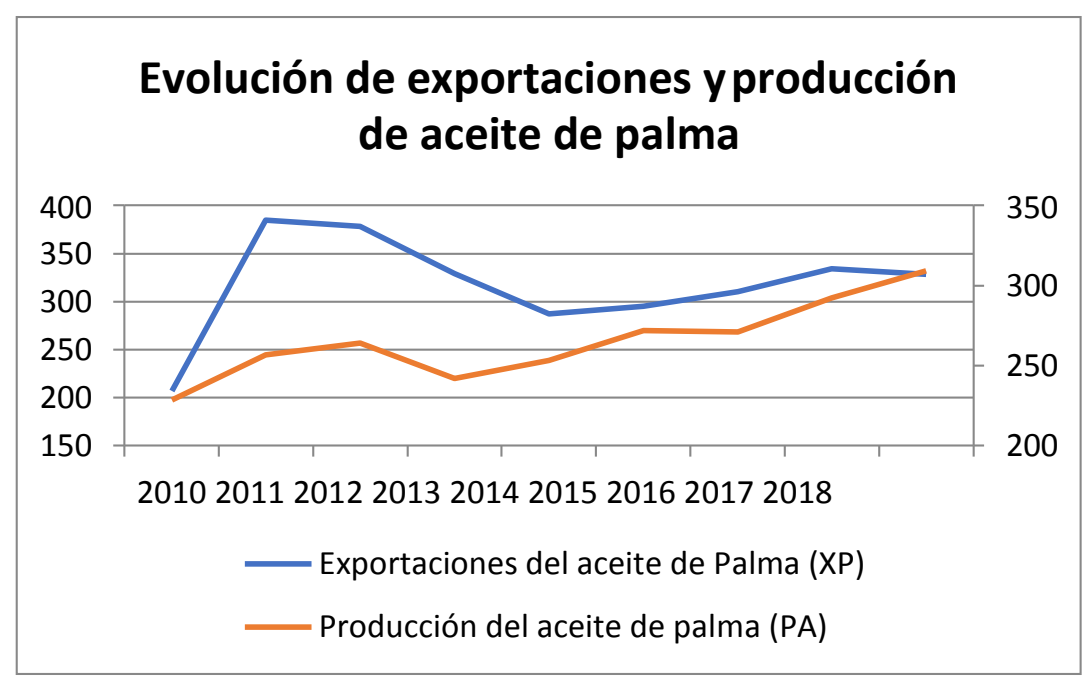

Este hecho revela que la producción del aceite de palma contribuye principalmente a la producción nacional y en menor mediad a las exportaciones. La evolución de este cultivo muestra el aporte positivo y constante a partir del año 2013.

\section{Evolución de las exportaciones de aceite de palma y producto interno agrícola}

Las exportaciones del aceite palma contribuyen en 0.160 de manera directa el desarrollo del Producto Interno Agrícola, el efecto es menor, debido a que la producción generada se utiliza principalmente para abastecer al mercado interno.

El crecimiento del Producto Interno Bruto agrícola ha sido consistente, pasando de 8 millones de USD en el 2010 a 11 millones en el 2018. 


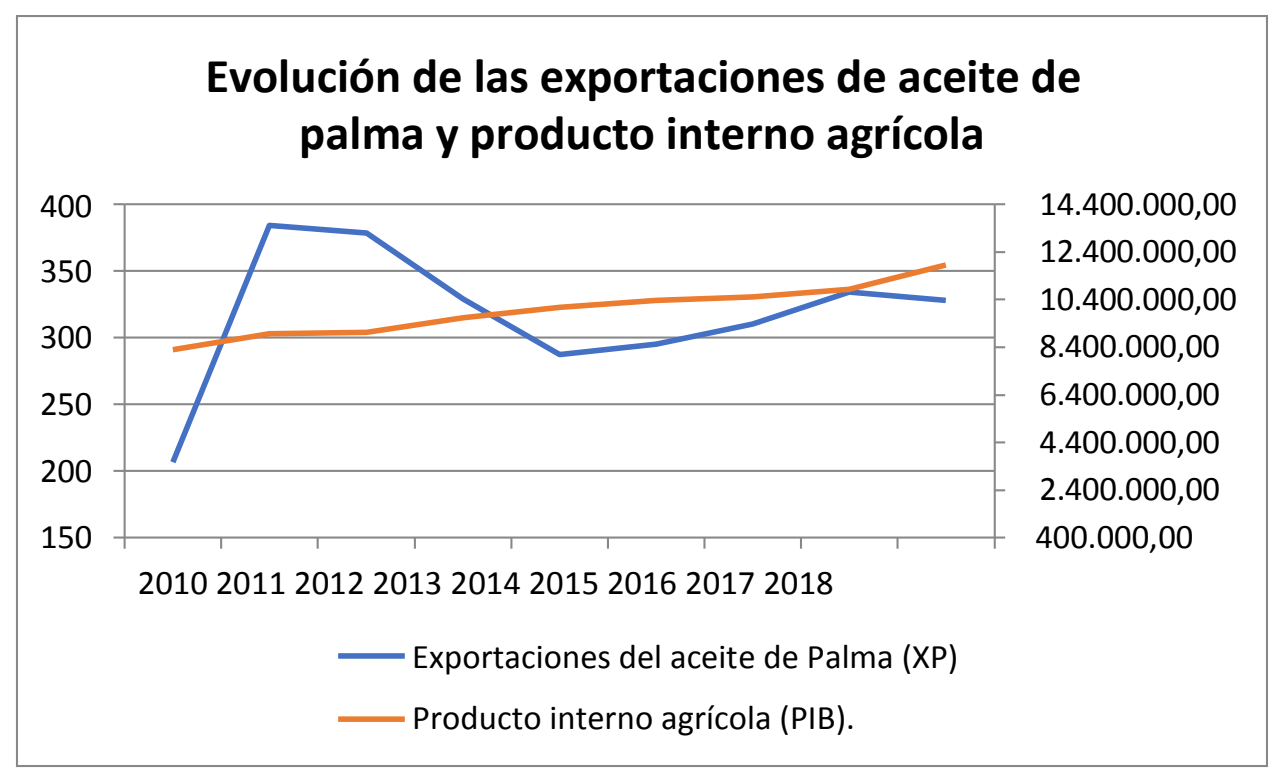

Como se observa en la evolución del producto interno bruto agrícola presenta un crecimiento positivo pese a la reducción del crecimiento del Producto interno bruto por los efectos de los fenómenos naturales. Los productos derivados han contribuido para el desarrollo productivo como el aceite de palmiste, al mantener la oferta para el sector industrial.

Se observa también que en el 2010 las exportaciones llegaron a 191.158 toneladas métricas (tn) lo cual representó ingresos por alrededor de 206.863.000 USD, y en el 2014 ascienden a 388.611 th el total de exportaciones. Este efecto equivale a un ingreso de alrededor de 310.121.000 USD. Lo que implica que en el 2010 una tn se exportó a un precio aproximado de 1.082 USD y en 2016 la tn alcanzó en 798 USD, lo que representa una reducción del 26,25\%.

\section{Evolución de la producción del aceite de palma y producto interno bruto agrícola}

La actividad económica agrícola relacionada a la palmicultura, constituye una dinamismo fundamental en la actividad agroindustrial, ya que estos contribuyen de manera directa en la oferta de empleos derivados de los diversos escalones del ciclo productivo de la producción del aceite de palma, desde su fase inicial, la siembre y cosecha; pasando por la industrialización para los productos derivados, hasta su comercialización final, que puede estar destinado a la producción nacional o exportación. De igual manera el uso del aceite de palma para la elaboración de biodiesel constituye un mercado de expansión. 
La producción del aceite de palma en el Ecuador permite obtener el aceite crudo de palma y el aceite crudo de palmiste. De estos se elaboran productos como: aceite de palmiste blanqueado, aceite de palma refinado, ácidos grasos destilados de palma, biodiesel de palma, entre otros relacionados y utilizados por la industria ecuatoriana para la fabricación de productos diversos que abarcan desde los cosméticos, textiles, productos de limpieza, a los alimenticios como los energéticos, e inclusive los de uso farmacéutico y cuidado de higiene personal.

Esta evolución constituye una producción creciente partir del año 2010 con ligeros decrecimientos en los períodos 2012 y 2016, cuyas afectaciones de debieron a la enfermedad en las plantaciones por el Cogollo.

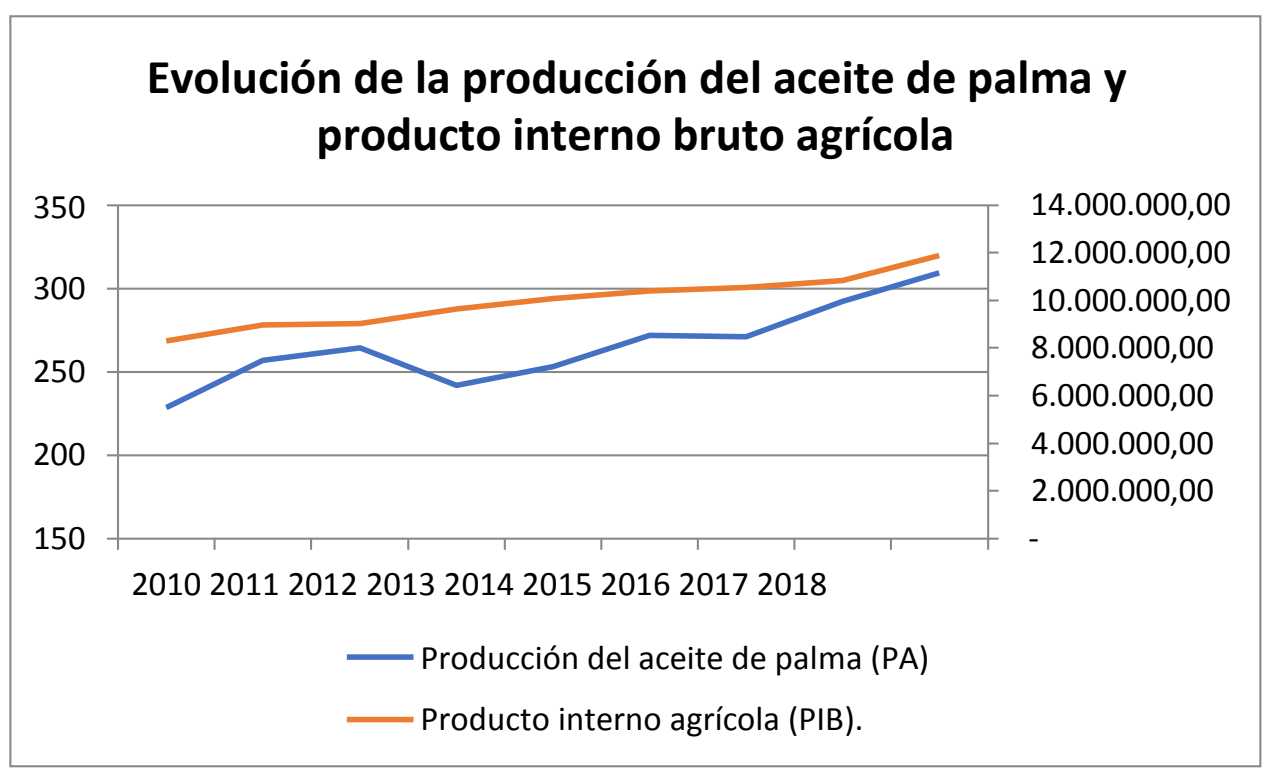

Se observa en el año 2011, se observa el crecimiento un crecimiento del $8 \%$ por el resultado de la implementación del Seguro Agrícola y la aplicación al subsidio de la urea, Estas medidas se aplicaron con el fin de proveer estabilidad en la producción, y lograr incrementar la productividad por hectárea.

En el 2012, presenta una desaceleración del 2\% en el crecimiento. Producto del efecto climático conocido como "la niña" el cual que afectó a los cultivos agrícolas. Para año el 2013 y 2014 se presentaron mejoras en la producción debido al mejor acceso de insumos, semillas mejoradas, trasferencia de tecnología. 


\section{Conclusiones}

La implementación de nuevos proyecto o emprendimientos, y el diversificar los bienes y servicios tradicionales para fomentar una educación especializada en la tecnificación, industrialización y mejor calidad del servicio en el sector, lo cual contribuirá en el 87,9\% en el crecimiento del PIBagrícola.

La Economía ecuatoriana, no es, sino la interacción de la administración conglomerada de los bienes y servicios de economía pública y de la empresarial privada, donde dentro de la nación interactúan los emprendimientos mercantiles, familiares, rurales, urbanos, del sector informal, el autoconsumo del trabajador asalariado, por medio de empresas, grupos económicos, holdings, empresas públicas, municipios y otros entes de tipo gubernamental.

Existe una relación fuerte y directa entre la producción del aceite de palma con el producto interno agrícola, lo que enmarcar la necesidad de reforzar el sector para afianzar los eslabones productivos que generen mayor valor agregado.

Existe una mediana relación positiva entre la producción del aceite de palma y las exportaciones, esto se debe principalmente a que parte de la producción se destina al consumo local.

Existe una débil relación positiva entre las exportaciones y el producto interno bruto agrícola, pero a pesar de ello constituye un rubro que genera valor al Producto Interno Bruto Nacional.

\section{Referencias Bibliográficas}

Agencia Ecuatoriana de Aseguramiento de la Calidad del Agro. (2014). AGROCALIDAD apoya al sector Palmicultor. Obtenido de: http://www.agrocalidad.gob.ec/agrocalidad-apoya-al-sectorpalmicultor/

Asociación Nacional de Cultivadores de Palma Aceitera. (2003). Manual Del Cultivo De Palma Aceitera. Quito, Ecuador: Pasquel Producciones Periodísticas.

Asociación Nacional de Cultivadores de Palma Aceitera. (2013). Investigaciones En Palma Aceitera. Quito, Ecuador: Grupo Impresor. 
Banco Central del Ecuador. (2010). Ecuador: Evolución de la Balanza Comercial. Ecuador. Obtenido de:

https://contenido.bce.fin.ec/documentos/Estadisticas/SectorExterno/BalanzaPagos/balanzaComercial /ebc201001.pdf

Banco Central del Ecuador. (2014). REPORTE DE COYUNTURA SECTOR AGROPECUARIO. Obtenido

https://contenido.bce.fin.ec/documentos/PublicacionesNotas/Catalogo/Encuestas/Coyuntura/Integra das/etc201304.pdf

Banco Central del Ecuador. (2016). Reporte de Coyuntura Sector Agropecuario. Ecuador. Obtenido de:

https://contenido.bce.fin.ec/documentos/PublicacionesNotas/Catalogo/Encuestas/Coyuntura/Integra das/etc201504.pdf

Banco Central del Ecuador. (2010-2016). Consulta de totales por NANDINA (toneladas y miles de dólares).

Obtenido

de:

https://www.bce.fin.ec/comercioExterior/comercio/consultaTotXNandinaConGrafico.jsp

Banco Central del Ecuador. (2017). REPORTE DE COYUNTURA SECTOR AGROPECUARIO.

Obtenido

de:

https://contenido.bce.fin.ec/documentos/PublicacionesNotas/Catalogo/Encuestas/Coyuntura/Integra das/etc201604.pdf

Carrión, L. y M. C. (1985). LA PALMA AFRICANA EN EL ECUADOR: Tecnología y Expansión Empresarial. Quito, Ecuador: FLACSO. Obtenido de: http://www.flacsoandes.edu.ec/libros/digital/45768.pdf

Matriz Productiva Ecuatoriana: Impacto del conocimiento y talento humano (2008-2016), Tapia y Viteri, 2107, CIDE, Ecuador

Instituto de Promoción de Exportaciones e Inversiones. (2014). Análisis Sectorial Aceite de Palma y Elaborados 2014. Obtenido de: http://www.proecuador.gob.ec/wpcontent/uploads/2015/05/PROEC_AS2016_ACEITEPALMA.pdf 
Instituto de Promoción de Exportaciones e Inversiones. (2016). PERFIL SECTORIAL DE AGROINDUSTRIA 2016. Obtenido de: http://www.proecuador.gob.ec/wpcontent/uploads/2016/04/PERFIL-AGROINDUSTRIA.pdf

Ministerio de Agricultura, Ganadería, Acuacultura y Pesca. (2014). Boletín Situacional Palma Aceitera $2014 . \quad$ Obtenido de: http://sinagap.agricultura.gob.ec/phocadownloadpap/cultivo/2014/lboletin-situacional-palmaaceitera-2014-actualizado.pdf

Ministerio de Comercio Exterior. (2017). Informe sobre el Sector Palmicultor Ecuatoriano mayo 2017. Obtenido de: http:/www.comercioexterior.gob.ec/wp-content/uploads/2017/09/informepalma-espa \%C3\%B1ol-.pdf.

Rincón, S. y Martínez, D. (2009). Análisis de las propiedades del aceite de palma en el desarrollo de $\mathrm{su}$ industria. Obtenido de: https://publicaciones.fedepalma.org/index.php/palmas/article/download/1432/1432

Sistema de Información Nacional de Agricultura, Ganadería, Acuacultura y Pesca. (2013). Boletín Situacional Palma $2013 . \quad$ Obtenido de: http://sinagap.agricultura.gob.ec/phocadownloadpap/cultivo/2013/palma_africana.pdf

Sistema de Información Nacional de Agricultura, Ganadería, Acuacultura y Pesca. (2014). Boletín Situacional Palma $2014 . \quad$ Obtenido de: http://sinagap.agricultura.gob.ec/phocadownloadpap/cultivo/2014/lboletin-situacional-palmaaceitera-2014-actualizado.pdf

Suárez, M. y Sabugo, A. (sf). ¿De dónde viene el aceite de palma? Obtenido de: http://www.aceitedepalma.org/origen

Tapia, C. (2014). "EXPORTACIÓN DE ACEITE DE PALMA AFRICANA DESDE SAN LORENZO PROVINCIA DE ESMERALDAS-ECUADOR HACIA EL MERCADO PARABRASIL". Obtenido de: http://dspace.udla.edu.ec/bitstream/33000/3757/1/UDLA-EC-TTEI-201405(S).pdf 


\section{Vallejo, Y. (2012). Diseño e Implementación de un Sistema de Costos en la Finca}

Ventura, S. (sf). Qué es la exportación. Obtenido de: https://www.gestion.org/estrategia-

empresarial/comercio-internacional/5666/que-es-la-exportacion/

Douglas Lind, W. M. (2012). Estadísticas aplicadas a los negocios y economía (Vol. 15). México: Mc Graw Hill.

Yánez, M. (2013). Estudio de factibilidad para la exportación de aceite de palma a India. Obtenido de: 